\title{
THE COMMUTATOR SUBGROUP OF A FREE TOPOLOGICAL GROUP NEED NOT BE PROJECTIVE
}

\author{
FRANCIS CLARKE
}

\begin{abstract}
It is shown that the commutator subgroup of the free topological group on the $n$-sphere is, for $n>1$, not projective and hence not free topological. The proof depends on the computation of the mod 2 cohomology ring of the classifying space of the commutator subgroup.
\end{abstract}

1. Introduction. The free topological group $F X$ on a pointed space $X$ was defined by Graev [3]. A subgroup of a free topological group need not be free topological, [3], [5], while [2] gives examples of subgroups of free topological groups which are not projective. The examples in [2] are not connected. We shall prove

THEOREM (1.1). Let $F S^{n}$ be the (Graev) free topological group on the n-sphere, and $C S^{n}$ its commutator subgroup. For $n>1, C S^{n}$ is not projective, and hence not free topological.

This gives examples of nonprojective subgroups which are closed and pathconnected.

The methods are homotopy theoretic. It is not known whether $C S^{1}$ is free; it is certainly isomorphic to a closed subgroup of $F S^{2}$ for which the inclusion is a homotopy equivalence, so homotopy theory cannot help further here.

I would like to thank R. Brown for raising the question.

2. Proof of Theorem (1.1).

Definition (2.1). The topological group $G$ is projective if given any diagram of morphisms of topological groups<smiles>[V][VH]</smiles>

such that $f$ has a continuous section, then $\psi$ lifts to a morphism $G \rightarrow H$.

Clearly a free topological group is projective.

Proposition (2.2). Let $G$ be a projective topological group which has the based homotopy type of a $C W$-complex and BG its classifying space. For any multiplicative cohomology theory $h^{*}$, all products in the ring $\tilde{h}^{*}(B G)$ vanish.

Received by the editors August 8, 1975 and, in revised form, November 6, 1975.

AMS (MOS) subject classifications (1970). Primary 22A99; Secondary 55F35.

Key words and phrases. Free topological group, commutator subgroup, projective topological group, Serre spectral sequence.

๑ American Mathematical Society 1976 
Proof. There are continuous homomorphisms $G \stackrel{s}{\rightarrow} F G \stackrel{f}{\rightarrow} G$ such that $f s=1_{G}$, where $f$ extends the identity $G \rightarrow G$, and the existence of $s$ follows from the projectivity of $G$. This implies that $B G$ is a retract of $B F G$. Since $G$ has the homotopy type of a CW-complex, $B F G$ has the homotopy type of the suspension $S G$ [4], [7]. The result follows; see, for example, Proposition 13.66 of [9].

If $X$ is the realisation of a simplicial set, as we may assume for $X=S^{n}, F X$ and $C X$ may be constructed simplicially; see [2]. If $A X$ denotes the free abelian group on $X$, there is an exact sequence $C X \rightarrow F X \rightarrow A X$.

It follows from the simplicial constructions that, up to homotopy, there is a fibration $A X \rightarrow B C X \rightarrow B F X$, to which we can apply the Serre spectral sequence; see [6, Chapter VI].

We will prove Theorem (1.1) by showing

Proposition (2.3). The mod 2 cohomology ring of $B C S^{n}$, for $n>1$, has a nontrivial multiplicative structure.

Proof. $A S^{n}$ is a $K(\mathbf{Z}, n)$, [1, Theorem 5.12], or [6, Theorem 24.5], and $B F S^{n}$ has the homotopy type of $S^{n+1}$.

The Serre spectral sequence

$$
H^{*}\left(S^{n+1} ; H^{*}(\mathbf{Z}, n ; \mathbf{Z} / 2)\right) \Rightarrow H^{*}\left(B C S^{n} ; \mathbf{Z} / 2\right)
$$

has $d_{n+1}$ as its only possible nonvanishing differential.

Let $x \in H^{n}(\mathbf{Z}, n ; \mathbf{Z} / 2)=E_{2}^{0, n}$ and $y \in H^{n+1}\left(S^{n+1} ; \mathbf{Z} / 2\right)=E_{2}^{n+1,0}$ be generators.

The homotopy exact sequence of the fibration shows that $B C S^{n}$ is $(n+1)$ connected, thus $d^{n+1} x=y$.

Recall that $H^{*}(\mathbf{Z}, n ; \mathbf{Z} / 2)$ is, for $n>1$, a polynomial algebra on the elements $\mathrm{Sq}^{I} x$, where $I$ ranges over all admissible sequences $\left(i_{1}, \ldots, i_{r}\right)$ of excess $\left\langle n\right.$ with $i_{r} \geqslant 2$ [8]. Since $x$ is transgressive so are all the $\mathrm{Sq}^{I} x$. It is then easy to see that the $E_{\infty}$-term of the spectral sequence is the tensor product of the exterior algebra on $x y$ with the polynomial algebra on the elements $x^{2}$ and $\mathrm{Sq}^{I} x$, for $I \neq(0)$. Hence the proposition is proved.

For $n=1$ the situation is quite different. $A S^{1} \rightarrow B C S^{1} \rightarrow B F S^{1}$ is, up to homotopy, the Hopf $S^{1}$-bundle over $S^{2}$.

\section{REFERENCES}

1. Ronald Brown, Cohomology with chains as coefficients, Proc. London Math. Soc. (3) 14 (1964), 545-565. MR 29 \#604.

2. (1975), 433-440.

3. M. I. Graev, Free topological groups, Izv. Akad. Nauk SSSR Ser. Mat. 12 (1948), 279-324; English transl., Amer. Math. Soc. Transl. (1) 8 (1962), 305-364. MR 10, 11.

4. J.P.L. Hardy and D. Puppe, Classifying spaces and universal categories (to appear).

5. D. C. Hunt and S. A. Morris, Free subgroups of free topological groups, Proc. Second Internat. Conf. Theory of Groups (Australian Nat. Univ., Canberra, 1973), Lecture Notes in Math., vol. 372, Springer-Verlag, Berlin and New York, 1974. pp. 377-387. MR 50 \# 4804.

6. J. P. May, Simplicial objects in algebraic topology, Van Nostrand Math. Studies, no. 11, Van Nostrand, Princeton, N.J., 1967. MR 36 \# 5942. 
7. J. W. Milnor, The construction FK, A Student's Guide to Algebraic Topology, by J. F. Adams, London Math. Soc. Lecture Notes, 4, 1972.

8. J.-P. Serre, Cohomologie modulo 2 des complexes d'Eilenberg-Mac Lane, Comment. Math. Helv. 27 (1953), 198-232. MR 15, 643.

9. R. M. Switzer, Algebraic topology-homotopy and homology, Springer-Verlag, Berlin and New York, 1975.

Department of Pure Mathematics, University College of Swansea, Wales, United KINGDOM 